\title{
Generation of transgene-free induced pluripotent stem cells from cardiac fibroblasts of goat embryos
}

\author{
Hanna $\mathrm{M}^{1,2}$, Sahito RGA ${ }^{1}$, Rateb $\mathrm{M}^{2}$, Kachiwal $\mathrm{AB}^{3}$, Seddiek $\mathrm{HA}^{2}$, Bhutto $\mathrm{B}^{4}$, Hescheler $\mathrm{J}^{1}$
}

\begin{abstract}
Induced pluripotent stem cells (iPSCs) hold a great potential for therapeutic regenerative medicine. The aim of this study was to generate induced pluripotent stem cells from goat embryonic cardiac tissue derived fibroblasts. The isolated cardiac fibroblasts from the cardiac tissue of goat embryos were positive for alfa smooth muscle actin, vimentin and discoidin domain receptor2. From these cells, we generated transgene free iPSCs using piggyBac transposons / transposase using five transcription factors (Oct4, Sox2, Klf, Myc and Lin 28). The generated iPSCs were SSEA1, SSEA4 and Oct4 positive. They were cultured on neofeeders using 20\% Serum replacement - IMDM with bFGF. They could form cystic and compact embryoid bodies that showed differentiated ectodermal and mesodermal like cells when cultured using 20\% FBS-IMDM without bFGF. The iPSCs, generated in the frame of this approach were produced without the use of integrating virus and the reprogramming transgenes were removed at the end of the process. Though there were limitations in the approach used, a substantial sign of reprogramming was obtained.
\end{abstract}

Key Words: PSCs; Pluripotency markers; Transcription factors; Cardiac fibroblast; Integration-Free nuclear reprogramming

\section{Introduction}

Generation of iPSCs is a highly meticulous procedure which has been introduced into the field of regenerative medicine using different methods. The pluripotent nature of embryonic stem cells (ESCs) with unlimited self-renewal abilities in culture made them the corner stone of regenerative medicine. However, there are major ethical concerns about ESCs as they are obtained from pluripotent cells of the inner cell mass from early-stage embryos. Therefore, it becomes difficult to generate patient-specific pluripotent stem cell lines ${ }^{[1]}$.

Takahashi and Yamanaka in $2006^{[2]}$ provided an answer to this ethical dilemma through the generation of induced pluripotent stem cells (iPSCs) thus avoiding the problematic questions concerning ESCs. iPSCs have a lot of therapeutic potentials and trials to generate patient-specific iPSCs from individuals suffering from different disorders have been attempted ${ }^{[3]}$.

The progression in iPSCs research and its application in the human therapy are still dependent on the generation of large animal models for the preclinical tests especially for cell transplantation due to their considerable anatomical and physiological similarities with humans ${ }^{[1]}$. Prior to the use of iPSC-derived cells or tissues in clinical application in human patients; their safety, integration and lack of tumorigenicity has to be investigated in proper animal models bearing significant similarities to humans ${ }^{[4]}$. Examples of such studies include those that were done on equine ${ }^{[5]}, \operatorname{pigs}^{[6]}$ and cattle ${ }^{[7]}$, each with a variable degree of success.

In 2011, Ren et al ${ }^{[8]}$ successfully generated iPSCs from primary ear fibroblasts of goats that showed morphologies similar to mouse embryonic stem cells and expressed SSEA1, Tra-1-60 and Tra-1-81 but were negative for SSEA-3 and SSEA-4. Goat iPSCs can thus be considered as having a lot of benefits as they gather the advantage that they are large animal-derived, related to an easily available domestic specimen, less expensive and so can be easily used for transplantation trials later.

Any actively dividing adult somatic cells can be reprogrammed. A lot of adult somatic cells can be used for generation of iPSCs such as fibroblasts ${ }^{[2,9]}$. The cardiac fibroblasts are drawing the attention nowadays as they have several fundamental and crucial functions in the cardiac tissue maintenance and regeneration ${ }^{[10]}$. So, reprogramming of cardiac fibroblasts ${ }^{[11]}$ may help to produce a stable well-developed cell line that can be differentiated to cardiomyocytes, which can be used efficiently in regenerative medicine.

Several methods were used for reprogramming such as retroviral delivery systems ${ }^{[2,9]}$ and that with minimal foot-print using retroand lentiviral vectors with loxP sites which serve as substrates for Cre-mediated excision of most of the integrated transgene. However, such methods leave genetic foot-print within the reprogramming cells which can result in complications such as the risk of tumorigenicity, mutations and considerably lower reprogramming efficiencies as in the case of retroviral transduction. Then, zero-foot-print methods have been introduced which include reprogramming by piggyBac, Sendai virus, episomal vectors, minicircles, adenovirus, direct microRNA transfection, and mRNA and protein overexpression of reprogramming factors ${ }^{[12]}$. The piggyBac transposon system is a reprogramming delivery method that has a lot of advantages which include having a very large cargo capacity and it does not leave "foot-print" mutations upon excision so it can generate transgene integration- and mutation-free iPSCs that can be used widely in regenerative medicine ${ }^{[13]}$.

Author Names in full: Mira Hanna ${ }^{1,2}$, Raja Ghazanfar Ali Sahito ${ }^{1}$, Moshira Rateb ${ }^{2}$, Allah Bux Kachiwal ${ }^{3}$, Hanan A. Seddiek ${ }^{2}$, Bachal Bhutto ${ }^{4}$, and Jürgen Hescheler ${ }^{1}$

${ }^{1}$ Institute of Neurophysiology, University of Cologne, Robert-Koch-Strasse 39, 50931 Cologne, Germany; ${ }^{2}$ Department of physiology, Faculty of medicine (Kasr El-Aini) Cairo University, El-Maniel, Cairo 11451, Egypt; ${ }^{3}$ Department of Veterinary Physiology and Biochemistry, Sindh Agriculture University Tandojam, Pakistan. ${ }^{4}$ Department of Veterinary Parasitology, Sindh Agriculture University Tandojam, Pakistan. 
The aim of this study was to generate transgene-free iPSCs from fibroblasts derived from cardiac tissue of goat embryos using the piggyback approach. The generated iPSCs were in vitro assayed for pluripotency.

\section{Materials and Methods:}

All applicable institutional guidelines of Neurophysiology Institute of University of Cologne and its partner institute Sindh Agriculture University, Tandojam, Pakistan for the care and use of animals were followed. Goat cardiac tissue samples were collected at the partner institute Sindh Agriculture University, Tandojam, Pakistan and tissue samples were shipped to Cologne. The isolation of cardiac fibroblast and generation of goat iPSCs were performed at institute of Neurophysiology University of Cologne, Germany.

\subsection{Isolation of goat cardiac fibroblasts (GCF)}

Cardiac tissue biopsy sample was provided by the collaborative partner University, Sindh Agriculture University, Tandojam. Generation of Goat iPSCs was performed at Institute of Neurophysiology University of Cologne, Germany. Pregnant goat, with two-month gestation period confirmed by ultrasonography, was selected with the permission of Institutional ethical committee Sindh Agriculture University, Tandojam, Pakistan and embryo/foetus was collected by the partner institute. Specimens from cardiac tissue of goat embryo were used to isolate cardiac fibroblasts according to the following protocol with minor modifications ${ }^{[14,15]}$. Myocardial tissue specimens were transferred in ice-cold phosphate buffered saline (PBS). Tissue samples were washed with Calcium-free Krebs-Ringer saline solution ${ }^{[16]}$. Cardiac tissue was put in the incubator for $15 \mathrm{~min}$ in Krebs-Ringer solution containing proteinase bacterial $(4 \mathrm{U} / \mathrm{ml})$ (Sigma Aldrich, P8038). Then the partially digested tissue was put in Krebs Ringer saline supplemented with a combination of collagenase $\mathrm{A}(1 \mathrm{mg} / \mathrm{ml}) \quad$ (Roche, Ref: 10103586001) and hyaluronidase $(0.5 \mathrm{mg} / \mathrm{ml})$ (Sigma Aldrich, H3506). All the enzymes were delivered as powder, prepared and stored in $-20^{\circ} \mathrm{C}$. The tissue was left in this solution for $20 \mathrm{~min}$ in the incubator at $37^{\circ} \mathrm{C}$ with gentle agitation. Lastly, the tissue was incubated with $1.0 \mathrm{mg} / \mathrm{ml}$ collagenase. Seven cycles for $20 \mathrm{~min}$ each at $37^{\circ} \mathrm{C}$ with gentle agitation were performed until complete digestion of the tissue. Every time the cells were harvested, centrifuged at $600 \mathrm{rpm}(\mathrm{RCF}=75 \mathrm{~g})$ for $4 \mathrm{~min}$ then the pellet was re-suspended in $1 \mathrm{ml} \mathrm{Ca}^{++}$free Krebs-Ringer solution and the $\mathrm{CaCl}_{2}$ was reintroduced incrementally at 4 min intervals with $3 \mu 1$ then $6 \mu 1$ followed by $9 \mu 1$ to reach a total of $18 \mu 1$ from a stock solution of 100 mMolar until the final calcium concentration reached 1.79 $\mathrm{mMol}$. The cells were centrifuged at $600 \mathrm{rpm}$ for $4 \mathrm{~min}$ then resuspended in $1 \mathrm{ml}$ culture medium and cultured in a $10 \mathrm{~cm}$ stem cell culture dish coated with $0.2 \%$ gelatin.

First medium changing was on day 3 and then every 2 days by Goat cardiac fibroblasts (GCF) culture medium. On Day 5 of culture, the GCFs were harvested by using trypsin $0.05 \%$. The cells were centrifuged at $1000 \mathrm{rpm}$ for 4 minutes and re-suspended in culture medium. Next, the cells were counted in a counting chamber (Hemocytometer) with trypan blue. Cells were sub-cultured on $6 \mathrm{~cm}$ gelatin coated plates at a density of 250,000 cells per plate. The Goat cardiac fibroblasts (GCF) culture medium used was $20 \%$ fetal bovine serum (FBS) (Sigma Aldrich) IMDM (Gibco by life technologies, 31980-022) with $0.1 \mathrm{mM}$ 2-Mercaptoethanol (Gibco, 31350-010), 1 $\%$ MEM NEAA (Gibco, 11140-035), 1\% (100U/ml) penicillin, (100 $\mu \mathrm{g} / \mathrm{ml}$ ) streptomycin (Gibco by life technologies, 15140-122) with $100 \mu \mathrm{g} / \mathrm{ml}$ Primocin (ampoule concentration $50 \mathrm{mg} / \mathrm{ml}$ purchased from Invivogen).

\subsection{Identification of cardiac fibroblasts}

a- Morphologically using light microscope (Details in supplementary data)

b- Using Immunocytochemistry for cardiac fibroblast cell markers ( $\alpha$ SMA, vimentin, and DDR2). The primary and secondary antibodies that were used, discussed in (Table 1) (Details in supplementary data).

\subsection{Reprogramming (induction of pluripotency)}

The process of Nucleofection of primary cardiac fibroblasts entails the following steps:

a. Cell preparation (details in supplementary data).

b. Culture of the cells after transfection

After transfection, the cells were cultured on 2 gelatine $0.2 \%$ coated $10 \mathrm{~cm}$ dishes. The cells were cultured using $15 \%$ FBS IMDM with $1 \%$ NEAA, $1 \%$ pen/strept and $0.1 \mathrm{mM}$ 2-Mercaptoethanol with addition of $6 \mathrm{ng} / \mathrm{ml}$ basic fibroblast growth factor $(\mathrm{bFGF})^{[18]}$. The cells were incubated and the medium was changed every day till day 4 after transfection. After day 4, every $10 \mathrm{~cm}$ plate was split to mitomycin $\mathrm{C}$ treated $\mathrm{MEF}$ seeded $6 \mathrm{~cm}$ plates while changing the medium to $20 \%$ knock out serum replacement (KOSR) IMDM with $1 \%$ MEM NEAA, $1 \%$ pen/strept and $0.1 \mathrm{mM} 2$-Mercaptoethanol with addition of $20 \mathrm{ng} / \mathrm{ml} \mathrm{bFGF}$. The culture medium was changed every day and the cells checked by light microscopy until tightly packed colonies with clear boundaries were recognized. The colonies were well recognized at day 12 after transfection. At day 14 after transfection, Geneticin $400 \mu \mathrm{g} / \mathrm{ml}$ was used and continued for 45 days for selection of the cells expressing the resistance under control of the UTF1 promoter, as the transfected cells should be geneticin resistant. UTF1-Neo (G418) is undifferentiated transcription factor1 driven antibiotic resistance in which G418 resistance driven by the undifferentiated transcription factor 1 (UTF1) promoter plus enhancer elements ${ }^{[19]}$. The reprogrammed goat cardiac fibroblasts with Transposons (T-GiPSCs) - potentially pluripotent cells - were mechanically split and passaged in clumps while they kept selfrenewal and proliferation forming large compact colonies that were more compact in the middle.

\section{c. Re-expression of transposase}

After stabilizing T-GiPSCs (the goat iPSCs with transposons), then the target was the re-expression of transposase (HypBase) for removal of the transposons achieving transgene-free GiPSCs. Several methods were attempted as the cells showed difficulty in transfection (the results were negative and not shown here).

Lipofectamine LTX and Plus Reagent was successfully used (purchased from Invitrogen, life technologies, Ref. 15338-100.). Protocol for 6 well plate (one well) was followed.

\section{d. Selection using Ganciclovir (Roche, CAS-82410-32-0):}

The reprogramming transposon is equipped with a thymidine kinase expression cassette allowing for negative selection. Therefore, cells that have successfully eliminated the reprogramming transposon will become resistant against ganciclovir, whereas cells that still express thymidine kinase from the reprogramming vector will be killed. 
Table 1: Antibodies used for immunocytochemistry to identify cardiac fibroblasts.

\begin{tabular}{|c|c|c|c|c|c|c|}
\hline $1^{\text {ry }}$ antibodies & Host & Isotype & Clone & Company & $\begin{array}{l}\text { Product } \\
\text { number }\end{array}$ & Dilution \\
\hline alfa Smooth muscle actin & Mouse & $\operatorname{IgG} 2 \mathrm{a}$ & $\begin{array}{l}1 \mathrm{~A} 4 \\
\text { monoclonal } \\
\text { antibody }\end{array}$ & Sigma & A5228 & $1: 500$ \\
\hline Vimentin & Mouse & $\operatorname{IgM}$ & $\begin{array}{l}\text { VIM-13.2 } \\
\text { monoclonal } \\
\text { antibody }\end{array}$ & Sigma & V5255 & $1: 200$ \\
\hline Discoidin Domain receptor 2 & Rabbit & $\operatorname{IgG}$ & polyclonal & Bioss & ABIN 755218 & $1: 200$ \\
\hline \multicolumn{7}{|l|}{$2^{\text {ry }}$ antibodies respectively } \\
\hline $\begin{array}{l}\text { IgG2a } \\
\text { (Alexa-fluor 647) }\end{array}$ & goat anti-mouse & & & $\begin{array}{l}\text { Life } \\
\text { Technologies }\end{array}$ & A21241 & $1: 1000$ \\
\hline $\begin{array}{l}\text { IgM } \\
\text { (Alexa-Fluor 555) }\end{array}$ & goat anti-mouse & & & $\begin{array}{l}\text { Life } \\
\text { Technologies }\end{array}$ & A21426 & $1: 1000$ \\
\hline $\begin{array}{l}\text { IgG }(H+L) \\
\text { (Alexa fluor 488) }\end{array}$ & goat anti-rabbit & & & $\begin{array}{l}\text { Life } \\
\text { Technologies }\end{array}$ & A11034 & $1: 1000$ \\
\hline
\end{tabular}

\subsection{Identification of iPSCs}

a- Alkaline Phosphatase staining: (Details in supplementary data).

b- ESC marker expression: The primary and secondary antibodies that were used, discussed in (Table 2) (Details in supplementary data).

c- Differentiation tests and trials of embryoid bodies generation: (Details in supplementary data).

\section{Results}

3.1. Isolation and characterization of Goat Cardiac Fibroblasts $(G C F)$

Goat cardiac fibroblasts were isolated according to the protocol discussed in the materials and methods and cultured on gelatincoated cell culture dishes with GCF culture medium. On Day 4, the cultured cells showed $70 \%$ confluency (Figure 1A). Usually 250,000 cells were seeded per $6 \mathrm{~cm}$ plate and increased 5 -folds during 5 days to become $1,250,000$ cells. GCFs were probed with antibodies for cardiac fibroblast markers $\alpha$ SMA, vimentin and DDR2 showing positive results (Figure 1B-D).

\subsection{Reprogramming of goat cardiac fibroblasts}

Expression of GFP, used as control to detect the effectiveness of nucleofection, was positively detected after 24 hours by using fluorescence microscopy (Figure 2A). On day 12, after transfection with the plasmid of interest, colony formation was recognized. Cells showed large nuclei with a few cytoplasm forming large compact colonies with defined borders. Figure 2 (B - F) shows the gradual appearance of the cells and T-GiPSCs colonies formation at different time points. The colonies were rose like compact large colonies with sharp borders. They appeared more compact in the center than in the periphery with occasional signs of spontaneous differentiation in the center region. T-GiPSCs were stable up to 60 passages (until the end of our experiment), in which the transfected cells were geneticinresistant.

3.3. Confirmation of pluripotency of reprogrammed cells with transposons (T-GiPSCS)

Alkaline phosphatase expression was assayed as a first indicator of successful reprogramming towards pluripotency in T-GiPSCs still bearing the reprogramming vector. The T-GiPSCs cells were positive for alkaline phosphatase as shown in Figure 3A.

Following positive detection of alkaline phosphatase, stage specific embryonic antigen-1 (SSEA1) and stage specific embryonic antigen4 (SSEA4) were analyzed as further pluripotency markers at that stage of the experiment. T-GiPSCs were positive to both SSEA1 and SSEA4 as detected by immunofluorescence staining (Figure 3B-D).

3.4. Generation and characterization of transgene-free goat iPSCs lines

The pmax GFP expression by goat iPSCs containing transposons (TGiPSCs) was successfully transfected using lipofectamine method Figure 4 (A \& B).

Then, Lipofectamine transfection reagent was used successfully to remove the transposons using Hypbase in which Figure 4C shows the morphology of the colonies that survived selection using ganciclovir after the removal of transposons. 
Table 2. Antibodies used for Immunohistochemistry to identify Pluripotency markers

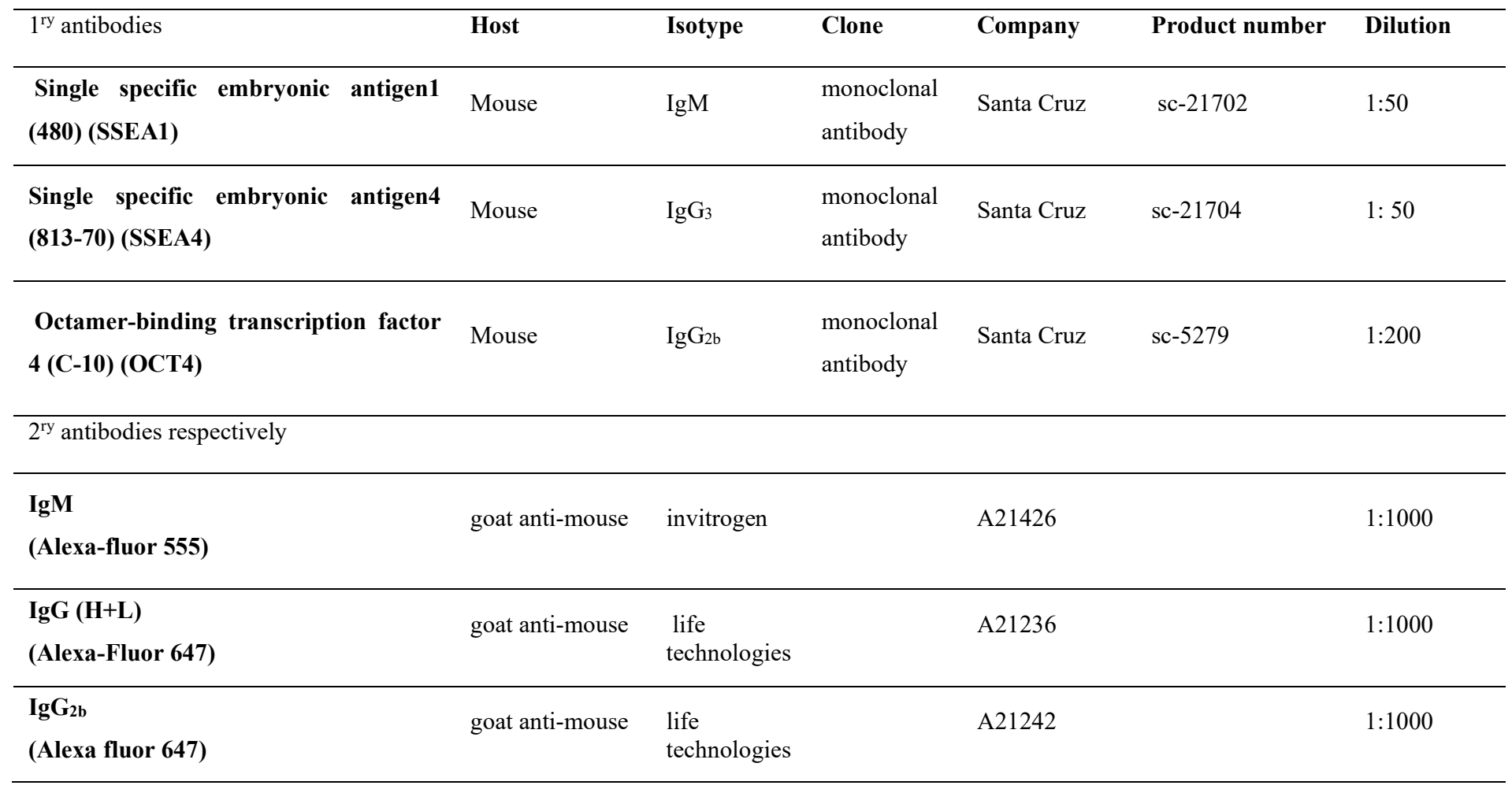
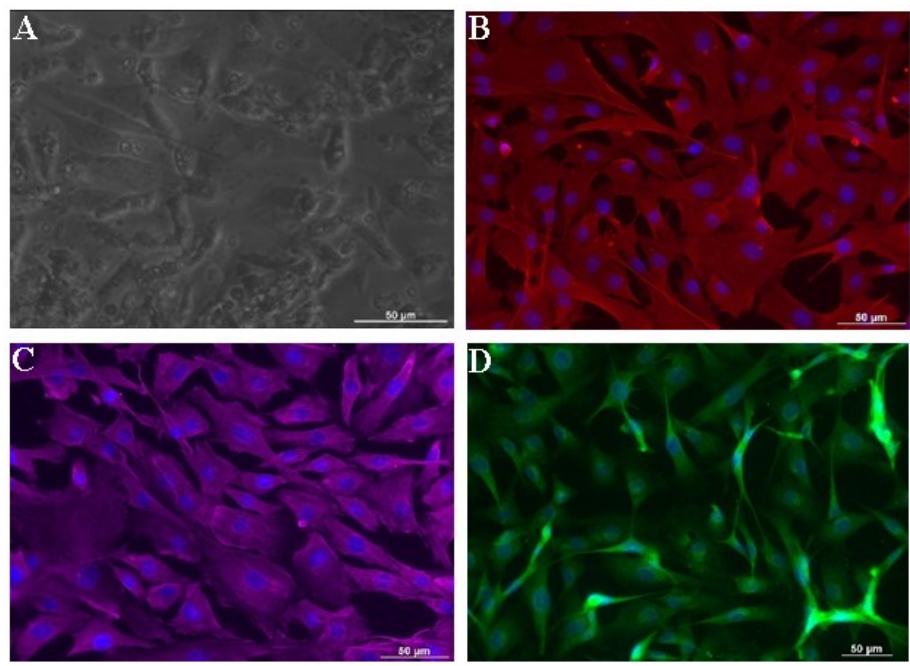

Figure $1(\boldsymbol{A}-\boldsymbol{D})$ : Isolation and characterization of goat cardiac fibroblasts: $(\boldsymbol{A})$ Phase contrast images of GCFs on day 4 after isolation using light microscope $10 x$ lens. GCFs were positive to anti $\alpha S M A(B)$, anti-vimentin $(\boldsymbol{C})$ and anti DDR2 (D) detected by fluorescence microscopy using $20 x L D$ lens.
Figure $2(\boldsymbol{A}-\boldsymbol{F}):(\boldsymbol{A})$ High power fields showing control for nucleofection with Pmax-GFP with extensive positive gene expression (green fluorescence) of GCFs. $(\boldsymbol{B}-\boldsymbol{F})$ Photomicrographs show the gradual appearance of the well-formed colonies in different time points using light microscopy and 10x lens: (B) Day 5 after transfection and DI after splitting and culture on murine embryonic feeders. (C) Day 7 after transfection and day 3 after splitting and culture on murine embryonic feeders. Until this step, the final morphology of the cells and the colonies were unknown. (D) Day 12 after transfection and D8 after splitting and culture on murine embryonic feeders. Arrows point to the borders of the arising colonies in formation. At this step, the final morphology of the cells and colonies could be recognized. (E) Day 15 after transfection and D11 after splitting and culture on murine embryonic feeders. The arrows point to the newly formed colonies. The colonies are well formed and ready for passage. (F) Photomicrograph of the well-formed colonies at day 5 of culture at Passage no.5 after mechanical splitting. The borders of the colonies are not apparent as the size of the colony was large.
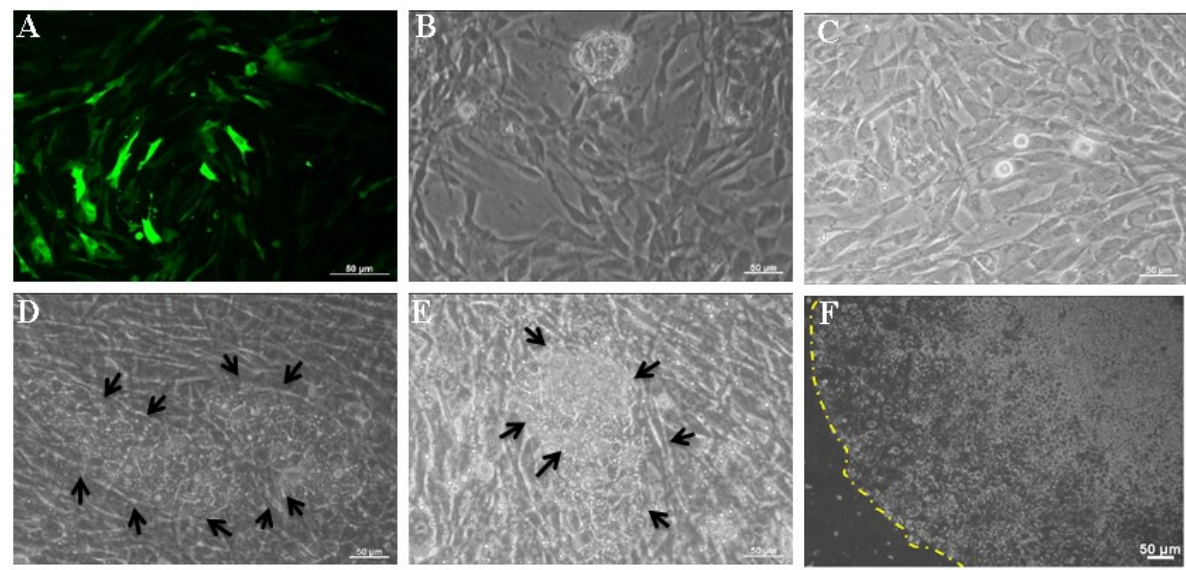

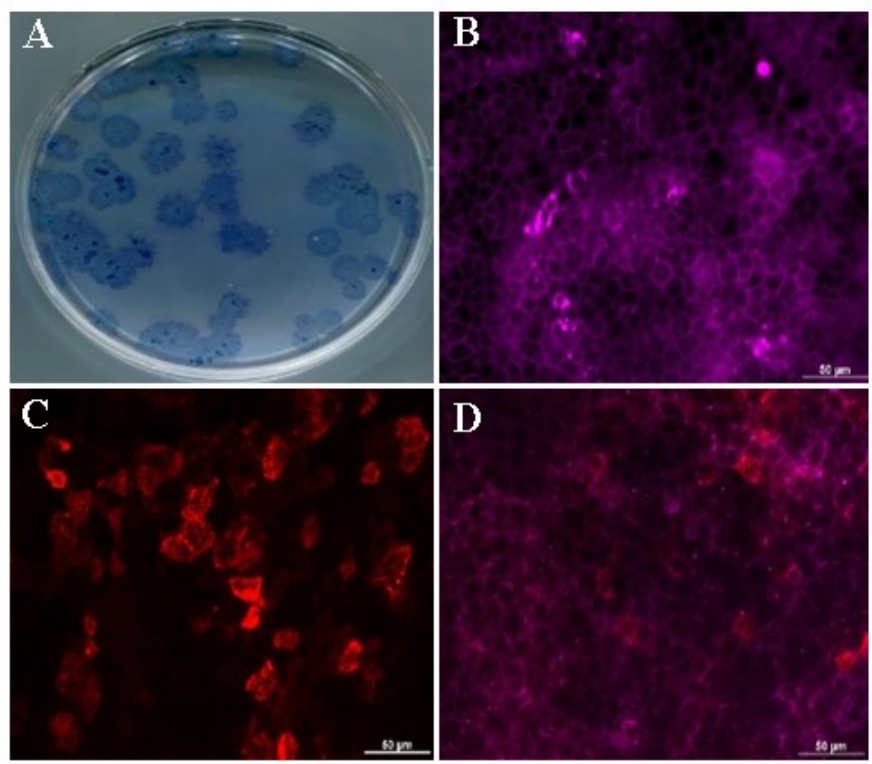

Figure $3(\boldsymbol{A}-\boldsymbol{D})$ : Characterization of the goat iPSCs before removal of the transposons (T-GiPSCs): (A) Picture of T-GiPSCs with transposons shows positive staining of alkaline phosphatase. Photomicrographs $(\boldsymbol{B} \& \boldsymbol{C})$ show T-GiPSCs that were positive to anti-SSEAI (purple) and positive to anti-SSEA4 (red) respectively. (D) Photomicrographs show double staining of T-GiPSCs with anti-SSEAI and anti-SSEA4 Pluripotency markers. The cells were positive to anti-SSEA1 (purple) and anti-SSEA4 (red) simultaneously. This can be seen in the photomicrograph of antiSSEA1 and SSEA4 that was detected also separately of the same colony in relation to their nuclei stained with DAPI (blue). The bright field shows the center of the colony. Photomicrographs $(\boldsymbol{B}-\boldsymbol{D})$ were taken using fluorescent microscope and $20 x L D$ lens.

Figure $4(\boldsymbol{A}-\boldsymbol{C})$ : Removal of transposons: $(\boldsymbol{A} \& \boldsymbol{B})$ Photomicrographs show Lipofectamine transfection reagent and pmax GFP of T-GiPSCs as control for re-expression of hypbase showing green fluorescent positive cells detected by fluorescence microscope 48 hours after transfection using $10 x$ Lens. (C) Photomicrograph shows GiPSCs colony after removal of the transposons keeping the same stemness characteristics; small cells with large nucleus, prominent nucleolus and small cytoplasm within large colonies using light microscope using $10 x$ lens.
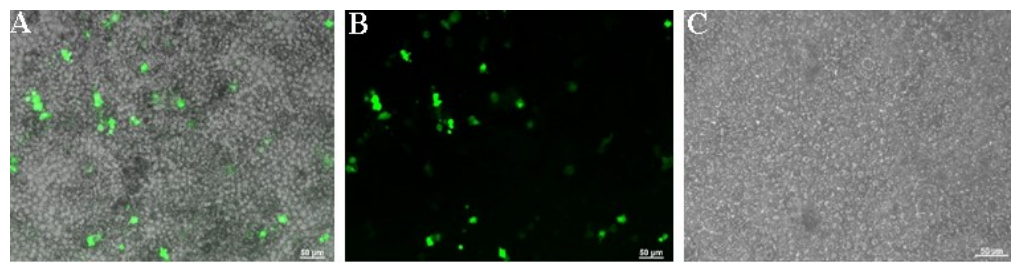

The GiPSCs (after removal of transposons) still show the same rose like compact large colonies with sharp borders. They appeared more compact in the center than in the periphery with occasional signs of spontaneous differentiation in the center region. The cells have large nuclei with small cytoplasm keeping self-renewal and proliferation through passages. After re-expression of transposase, the isolated transposon-free iPSCs (GiPSCs) were ganciclovir-resistant. GiPSCs was characterized as being stable, capable of self-renewal and proliferation, forming well defined compact colonies and maintaining pluripotency for 8 passages (we then froze the cell line for further experiments).

The GiPSCs were positive for alkaline phosphatase after the removal of the transposons as an indication of keeping their pluripotency (Fig.5A). The immunocytochemistry tests were repeated to detect the pluripotency markers SSEA1 and SSEA4 after removal of the transposons in which the G-iPSCs were remained positive for both after the transposons removal (Figure 5B-D).
In addition, the nuclear marker OCT4 was used to test for pluripotency after removal of the transposons and all cells were positive (Figure 5E \& F).

\subsection{Trials of embryoid body (EB) generation as a form of differentiation}

The differentiation was performed in EB model in which cystic EBs were generated from clumps shown in (Figure 6A) and compact EBs were generated from single cells displayed in (Figure 6B) "as described in detail in the material and methods section". Figure $6 \mathrm{C}-$ F) show morphological signs of differentiation of the cultured cystic EBs cultured on fibronectin. The different morphological structures of differentiated stem cells include: polyhedral epithelial like cells denoting ectodermal differentiation, spindle-shaped cells denoting mesenchymal differentiation and also stellate-shaped cells having large cell body with long cytoplasmic processes indicating a neuronal-like structure.
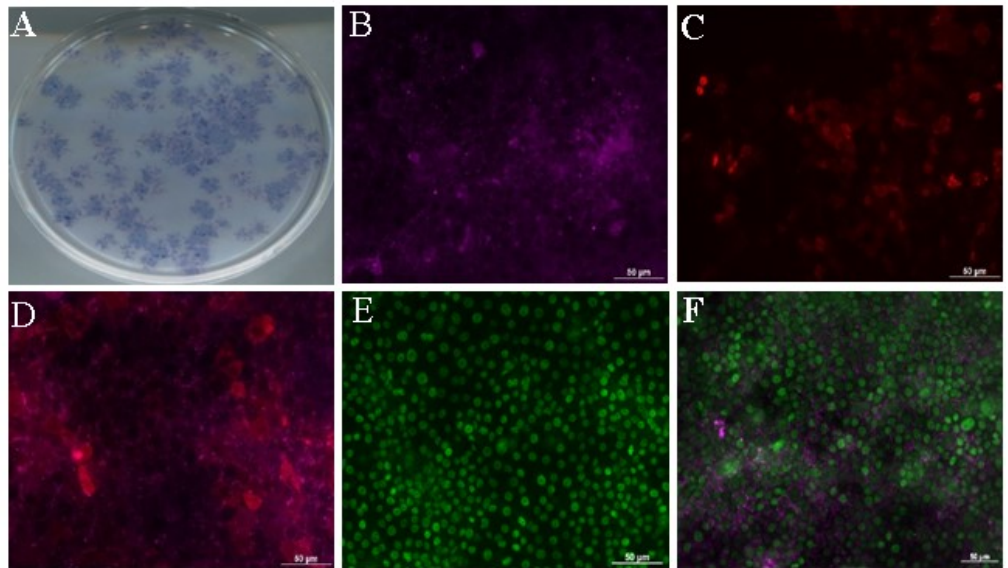

Figure $5(A-F)$ : Characterization of GiPSCs (after removal of transposons): (A) Picture of GiPSCs with transposons shows positive staining of alkaline phosphatase. Some of the colonies have not well stained borders indicating some differentiation in the border of the colonies that may have occured. Photomicrographs $(\boldsymbol{B} \& \boldsymbol{C})$ show the GiPSCs in which they were positive to anti-SSEA1 (purple) and anti-SSEA4 (red) respectively. (D) Photomicrographs show double staining of GiPSCs with anti-SSEAI and antiSSEA4 Pluripotency markers. The cells were positive to antiSSEAI (purple) and anti-SSEA4 (red) simultaneously. (E) Photomicrographs show anti-Oct4 Pluripotency marker of GiPSCs in which they were all positive to Oct4. (F) Photomicrographs show double staining of the GiPSCs with anti-SSEAI (purple) and anti-Oct4 (green) Pluripotency Markers in which they were positive to SSEAI and Oct4 simultaneously. Photomicrographs $(\boldsymbol{B}-\boldsymbol{F})$ were taken using fluorescent microscope and $20 x L D$ lens. 

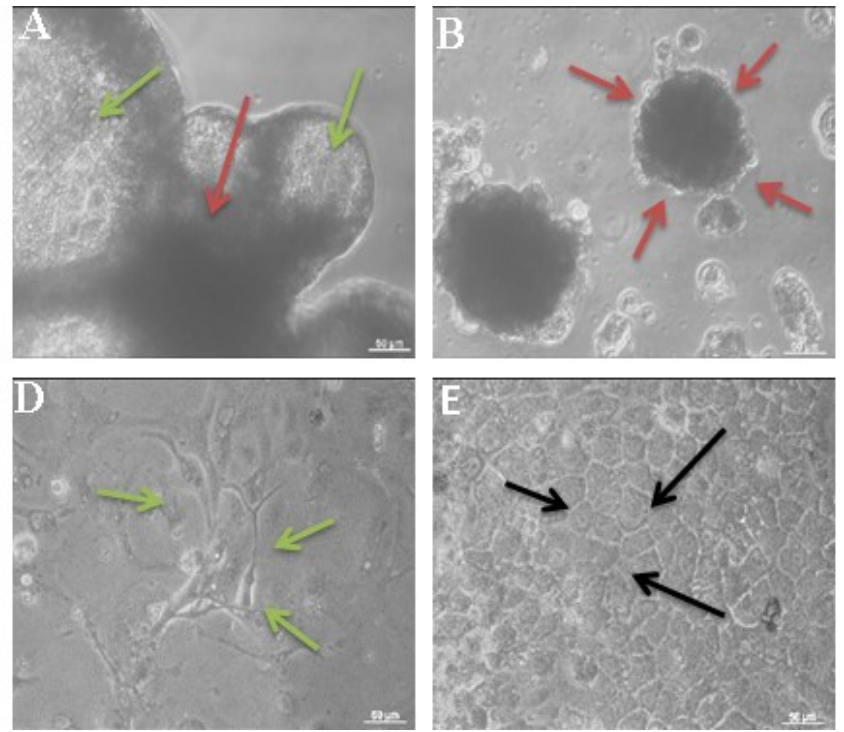
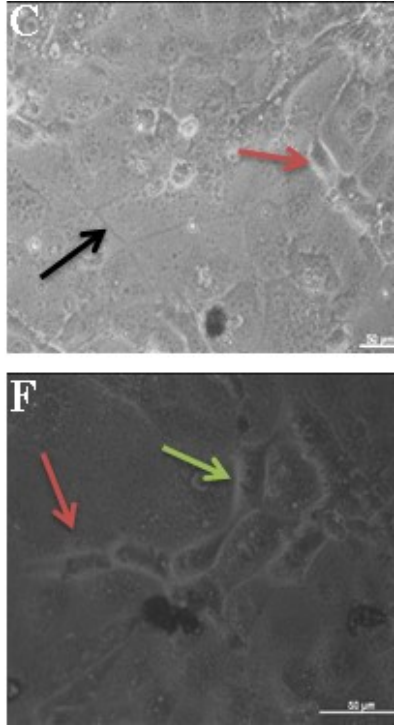

Figure $6(\boldsymbol{A}-\boldsymbol{F})$ : Cystic and compact EBs generation and culture: (A) Photomicrograph shows cystic EBs generated by using clumps of cells. The green arrows show the cystic part of $E B s$ and the red arrows show the compact part. Photomicrograph (B) show generated compact EBs using single cells in day 5. The red arrows show the borders of the compact EB. $(\boldsymbol{C}-\boldsymbol{F})$ Photomicrographs show cultured cystic EBs on fibronectin coated plates after 7 days. They show different morphological structures with large cytoplasm in relation to the nucleus that indicate differentiation to different cell types. They show differentiated stem cells with different morphological structure: polyhedral epithelial like cells denoting ectodermal differentiation (black arrow), spindle shaped cells denoting mesenchymal differentiation (red arrow) and also stellate shaped cells having large cell body with long cytoplasmic processes neuronal like structure (green arrow). All photomicrographs were examined under light microscope using $10 x$ lenses.

\section{Discussion}

The study describes here a trial of reprogramming of goat cardiac fibroblasts $(\mathrm{GCFs})^{[11]}$ isolated from cardiac embryonic tissue using a transgene-free approach of piggyBac transposition to generate transgene-free goat induced pluripotent stem cells (GiPSCs).

Fibroblasts are phenotypically diverse and experimental evidence has indicated that considerable heterogeneity exists between fibroblasts from different tissues ${ }^{[20]}$. This phenotypic plasticity has created a unique challenge in attempts to better define the cardiac fibroblast ${ }^{[21]}$. In the present study, isolated GCFs were positive for 3 markers: aSMA, vimentin and DDR2 to confirm their phenotype.

DDR2 and vimentin have been identified as specific markers for fibroblasts ${ }^{[21,22,23]}$. $\alpha$ SMA is a controversial marker as some studies report its absence from the normal cardiac fibroblasts, minimal or upregulated expression due to the stressful conditions in the surrounding physiological (in-vivo) or cultural (in vitro) environment ${ }^{[23]}$. Also, the occasional detection of $\alpha$-SMA fibers did not allow terming these cells as myofibroblasts ${ }^{[22]}$.

Cardiac fibroblasts are a good source of somatic cells for generation of iPSCs that later can be used for differentiation to cardiomyocytes ${ }^{[24]}$. We succeeded with five defined factors using Lin 28 in addition to the Yamanaka reprogramming factors OCt4, SOX2, $\mathrm{KLF} 4$, and c-MYC (OSKML) in the induction and maintenance of pluripotency in the cardiac fibroblasts. The goat iPSCs before removal of the transposons (T-GiPSCs) were stable up to 60 passages, in which the transfected cells are geneticin resistant. After re-expression of transposase, the isolated transposon-free iPSCs (GiPSCs) were Ganciclovir resistant. GiPSCs was characterized as being stable, capable of self-renewal and proliferation, formed well defined compact colonies and maintained pluripotency for 8 passages.

Few studies were done on goat cells as it has been reported that Goat iPSCs generation is as difficult as isolation and culture maintenance of goat ESCs. Ren et al, 2011 ${ }^{[8]}$ reported difficulties in reprogramming goat primary ear fibroblasts. They could not generate goat iPSCs either by using lentivirus transduction encoding cDNAs of Oct4, Sox2, cMyc, and Klf4 or by adding viruses encoding Nanog and Lin28 genes to the infection cocktail. However, the SV40 large T antigen and the catalytic subunit of human telomerase (hTERT) using a drug-inducible lentivirus encoding OSCKNL improved significantly the efficiency of iPSCs generation though withdrawal of doxycycline resulted in transgene silencing and loss of pluripotency.
In our study, generation of goat iPSCs could be easily produced from cardiac fibroblasts without adding chemical inhibitors. This is in line with the study done by Song et al $2013^{[18]}$ as they generated goat iPSCs from goat primary ear fibroblasts using lentivirus encoding only four human transcription factors, OCT4, SOX2, KLF4, and CMYC without adding chemical inhibitors. However, in our study, the five previously mentioned factors (OSKML) were used ${ }^{[13]}$. Lin 28 was chosen to be added to the OSKM in this study as it was reported by $\mathrm{Yu}$ et al, $2007^{[25]}$ that Lin 28 was considered an essential factor. It enhanced the reprogramming when added to their cocktail containing Oct4, Sox 2 and Nanog. On the other hand, Song et al 2013 $3^{[18]}$ reported that these goat iPSCs were forming colonies with distinct boundaries similar to those of human ESCs and iPSCs. However, they stated that the undifferentiated phenotype of their goat iPSCs was labile before passage 5. Sandmaier et al, 2015 ${ }^{[26]}$ reported the generation of goat iPSCs using forced expression of bovine POU5F1, SOX2, MYC, KLF4, LIN-28, and NANOG reprogramming factors in combination with a MIR302/367 cluster, delivered by lentiviral vectors.

Generation of iPSCs that can be used for regenerative medicine requires a stable, safe and non-mutated genome. The integration free - generated iPSCs can be considered as ideal when compared to other techniques such as retroviral transfection, which despite its high efficiency shows mutations and tumor formation, and transient transfection as adenovirus and repeated plasmid transfection show very low transfection efficiency ${ }^{[2,9,25]}$. Loh et al 2012 $2^{[27]}$ used Crerecombinase LoxP, which is also used for excision of the already integrated genome inserted using retrovirus transfection, however, residual vector sequences, might still be left behind and cause insertional mutations.

In our study, goat cardiac fibroblasts could be easily reprogrammed using five defined factors and piggyBac transposons with $2 \mathrm{~A}$ peptide system that serves as efficient vehicle for large cargos even more than five factors and depending on the excision of the already integrated transposons (transgene free). Yusa and his colleagues in $2009^{[13]}$ showed that the five known factors (Oct4, Sox2, Klf4, cMyc and Lin28) were successfully combined into a single vector that induced reprogramming of fibroblasts more efficiently when compared to the four factors vector though the cells they used were murine embryonic feeder. On the other hand, other studies as that done by Kaji et al, 2009 and Woltjen et al 2009 ${ }^{[28,29]}$ reported using piggyBac transposons and four factors (OSKM) for successfully reprogramming human and mouse embryonic fibroblasts to iPSCs. Mitra et al, 2008 ${ }^{[30]}$ supported the suggestion that the piggybac 
transposons/transposase system that was used in our study is safe for producing GiPSCs cell lines with clean excision sites leaving no mutations behind. However, for a final proof it would be necessary to analyze the number of integrated transposons prior to excision, map their location and perform sequence analysis after integration in comparison to unmodified cells. Given the fact that the cells generated in this study were not intended for clinical use; this step was omitted.

GiPSCs generated were alkaline phosphatase positive, Oct4 positive and interestingly; they were SSEA1 and SSEA4 positive. Some studies have shown expression of both SSEA1 and SSEA4 by iPSCs as we found while other studies have suggested a degree of differentiation when they are expressed simultaneously. The morphology and the expression of the SSEA antigens of bovine iPSCs generated by Lin et al $2014^{[31]}$ resembled those of mouse ESCs/iPSCs, rather than those of human ESCs/iPSCs but in addition to SSEA1, they found a strong signal for SSEA4. Han et $a l, 2011^{[7]}$ produced bovine iPSCs that were positive for SSEA1 but are weakly positive for SSEA4. Sumer et al, 2011 ${ }^{[32]}$ showed that bovine iPSCs derived from adult ear fibroblast were positive to SSEA1 and SSEA4 using five factors OSKM and Nanog. In rabbit iPSCs, Osteil et al, 2013 ${ }^{[33]}$ generated 3 lines of iPSCs and isolated 4 lines of ESCs with different expression of SSEA markers. The 3 iPSC lines showed heterogeneous expression of SSEA1 and SSEA4 demonstrating 4 cell subpopulations, while all the 4 lines of ESCs expressed only SSEA-1, despite that both the rabbit ESCs and iPSCs needed bFGF for their culture. Yan et al, 2008 ${ }^{[34]}$ have isolated goat embryonic stem cells with colonies that maintained undifferentiation for 18 passages. The goat ESCs expressed the proteins Nanog, Oct4 and SSEA-3, whereas the protein of SSEA-4 was absent and the protein of SSEA-1 was weakly expressed.

One suggested explanation for the finding of mosaic SSEA4 expression is the hypothesis that there is a process of differentiation in some cells initiated within the colonies. This finding could point to a need for further fine-tuning of the culture conditions. In comparison, it was stated that the differentiating human PSCs showed upregulation of SSEA1 expression ${ }^{[35]}$ especially when they differentiate to multipotent cardiac progenitors, which have the ability to differentiate into Cardiomyocytes, smooth muscle cells, and endothelial cells ${ }^{[36]}$. However, Brimble et al, 2007 ${ }^{[37]}$ showed that in absence of SSEA-3 and SSEA-4, human ESCs remained pluripotent which was proved by their ability of self-renewal, three germ layer differentiation in vitro and teratoma formation.

Another possible explanation, is that these goat iPSCs showed an intermediate state between naiive and primed pattern of iPSCs. Naiive PSCs are similar to murine ESCs and express SSEA1 while primed PSCs are similar to human and express SSEA4. The naiive versus primed state of iPSCs are molecularly and functionally different, however, both types express genes of pluripotency ${ }^{[38]}$. In addition, both types can differentiate into three germ layers (ectoderm, mesoderm, and endoderm) in vitro ${ }^{[39]}$. The major difference between them is their ability to develop chimeric offspring. Naiive cells can contribute to the development of chimeras while primed PSCs cannot ${ }^{[40]}$.

In our study, the pluripotency of the GiPSCs was maintained using bFGF as done by Song and his colleagues $2013^{[18]}$ as well as other studies done on human PSCs and nonhuman primate PSCs ${ }^{[41,42,43]}$. In addition, to maintain the characteristic stemness of GiPSCs, we cultured them with mitomycin-C-treated murine embryonic fibroblasts (MEFs), which were used as feeder cells. In the absence of MEFs, the stemness features were lost. Lin et al, $2014^{[31]}$ detected the same in which the MEFs were important for maintaining stemness state of iPSCs but they were using BMP4 as a supplement in culture medium instead of the bFGF. Huang et al, $2011^{[44]}$ reported bovine iPSCs that were cultured without feeders in a chemically defined medium containing LIF and two inhibitors of MEK1/2 and glycogen synthase kinase-3 signaling. It is, thus, clear that the conditions needed to maintain pluripotency in the culture medium are still an area that require more experimental research to ensure needed results are achieved.

Based on our results, GiPSCs needed to be dispersed into small clumps, not single cells, to be able to form colonies; otherwise most of the single cells will not form colonies and tend to differentiate or undergo cellular death. This result is in line by what was reported by Song et al, $2013^{[18]}$ who stated that their cells should be passaged mechanically into clumps to minimize the propagation of differentiated cells. In addition, Our GiPSCs also could not tolerate single cell dissociation by trypsin showing cellular death. In this aspect it is similar to human PSCs that are vulnerable to apoptosis upon cellular detachment and dissociation ${ }^{[45]}$.

In our study, GiPSCs could generate two types of embryoid bodies (EBs): cystic and compact EBs. The same result was shown by Müller et al, 2009 ${ }^{[46]}$ but using monkey ESCs. Compact EBs resemble the morula embryonic stage ${ }^{[47]}$. While, Cystic EBs resemble the blastocyst embryonic stage morphologically (develop a fluid filled central cavity like that of a blastocyst with differentiation of a columnar epithelium with a basal lamina) ${ }^{[48,49]}$. Our cystic embryoid bodies attached better on fibronectin coated surfaces than normal tissue culture dishes, and that was in line with the study done by Sivasubramaiyan et al, 2009 ${ }^{[50]}$.

From the previous discussion, we can conclude that:

Good quality goat cardiac fibroblasts (GCFs) can be easily isolated and cultured on gelatin-coated plates.

Discoidin doman receptor 2 (DDR2) is one of the important and specific factors to characterize the cardiac goat fibroblasts beside vimentin and $\alpha$ smooth muscle actin.

Goat cardiac fibroblasts are easily reprogrammed using nucleofection generating goat induced pluripotent stem cells containing transposons (T-GiPSCs).

Using piggyBack transposons/transposase and five transcription factors (Oct4, Sox2, Klf4, c-Myc and Lin28) is a good reprogramming method to generate transgene free iPSCs from GCFs.

Lipofectamine technique was a good technique used for transfection of the GiPSCs in culture to remove the transposons.

The GiPSCs, which were generated in our study, were SSEA1, SSEA4 and Oct4 positive.

The GiPSCs were cultured on neofeeders using 20\% Serum replacement - IMDM with bFGF showing large compact colonies with well-defined borders and central differentiation. They passaged mechanically in clumps.

They could form compact and cystic embryoid bodies that showed differentiated ectodermal and mesodermal like cells when cultured using 20\% FBS-IMDM without bFGF. 


\section{Outlook and future experiments}

Further work is needed to:

- Adjust the culture conditions for the best physiological environment needed by this new cell line to maintain pluripotency.

- Include a panel of markers that can positively identify the capacity of these cells to differentiate into three germ layer cell types with a capacity for teratoma formation.

- Induction of differentiation of GiPSCs to cardiomyocytes in order to compare between the physiological efficiency of the GiPSCs Cardiomyocyte derived from cardiac fibroblasts and that derived from skin adult fibroblast.

\section{Limitations and challenges}

Available literature is very limited as regards goats as a source of pluripotent cells.

Removal of the transposons proved to be one of the very difficult steps in this work.

\section{References}

1. Chronowska E. Induced pluripotent stem (iPS) cells in domestic animals - recent achievements - a review. Animal Science Papers and Reports. 2013; 31 (2): 89 - 99.

2. Takahashi K, Yamanaka S. Induction of Pluripotent Stem Cells from Mouse Embryonic and Adult Fibroblast Cultures by Defined Factors. Cell. 2006; 126 (4): 663 - 76.

3. Singh VK, Kalsan M, Kumar N, Saini A, and Chandra R. Induced pluripotent stem cells: applications in regenerative medicine, disease modeling, and drug discovery. Front Cell Dev Biol. 2015 3: 2 .

4. Nowak-Imialek M, Kues W, Carnwath JW, Niemann H. Pluripotent stem cells and reprogrammed cells in farm animals. Microscopy and Microanalysis, 2011; 17 (4): 474 - 97.

5. Nagy K, Sung HK, Zhang P, Laflamme S, Vincent P, AghaMohammadi S, Woltjen K, Monetti C, Michael IP, Smith LC, Nagy A. Induced pluripotent stem cell lines derived from equine fibroblasts. Stem Cell Rev. 2011; 7 (3): 693 - 702.

6. Wu Z, Chen J, Ren J, Bao L, Liao J, Cui C, Rao L, Li H, Gu Y, Da $\mathrm{H}$, Zhu H, Teng X, Cheng L, Xiao L. Generation of pig induced pluripotent stem cells with a drug inducible system. J. Mol. Cell. Biol. 2009; 1(1): $46-54$

7. Han X, Han J, Ding F, Cao S, Lim SS, Dai Y, Zhang R, Zhang Y, Lim B, Li N. Generation of induced pluripotent stem cells from bovine embryonic fibroblast cells. Cell research. 2011; 21 (10): $1509-12$

8. Ren J, Pak Y, He L, Qian L, Gu Y, Li H, Rao L, Liao J, Cui C, Xu X, Zhou J, Ri H, Xiao L. Generation of hircine-induced pluripoten stem cells by somatic cell reprogramming. Cell research. 2011;21 (5): 849 - 53

9. Takahashi K, Tanabe K, Ohnuki M, Narita M, Ichisaka T, Tomoda $\mathrm{K}$, Yamanaka S. Induction of pluripotent stem cells from adult human fibroblasts by defined factors. Cell. 2007; 131 (15): $861-$ 72.

10. Furtado MB, Costa MW, Pranoto EA, Salimova E, Pinto A, Lam NT, Park A, Snider P, Chandran A, Harvey RP, Boyd R, Conway SJ, Pearson J, Kaye DM, Rosenthal NA. Cardiogenic Genes Expressed in Cardiac Fibroblasts Contribute to Heart Development and Repair. Circ. Res. 2014; 114 (9): 1422 - 34

11. Jiang B, Dong H, Li Q, Yu Y, Zhang Z, Zhang Y, Wang G, Zhang Z. Differentiation of Reprogrammed Mouse Cardiac Fibroblasts into Functional Cardiomyocytes. Cell Biochem Biophys. 2013; 66 (2): $309-18$.

12. Rao MS, Malik N. Assessing iPSC Reprogramming Methods for their Suitability in Translational Medicine. J Cell Biochem. 2012; 113 (10): $3061-68$

13. Yusa K, Rad R, Takeda J, Bradley A. Generation of transgene-free induced pluripotent mouse stem cells by the piggyBac transposon. Nat Methods. 2009; 6 (5): 363 - 69

14. Bird S, Doevendans P, van Rooijen M, Brutel de la Riviere A, Hassink R, Passier R, Mummery C. The human adult cardiomyocyte phenotype. Cardiovascular Research. 2003; 58 (2) $423-34$.

15. Peeters G, Sanguinetti M, Eki Y, Konarzewska H, Renlund D, Karwande S, Barry W. Method for Isolation of human ventricular myocytes from single endocardial and epicardial biopies. Am J Physiol Heart Circ Physiol. 1995; 268 (4): H1757 - 64

16. Bird SD, Walker RJ, Hubbard MJ. Altered free calcium transients in pig kidney cells (LLC-PK1) cultured with penicillin streptomycin. In Vitro Cell Dev Biol Anim. 1994; 30 (7): 420 24.

17. Yusa K, Zhoub L, Lia MA, Bradleya A, Craig NL. A hyperactive piggyBac transposase for mammalian applications. PNAS. 2011 108 (4): $1531-36$

18. Song H, Li H, Huang M, Xu D, Gu C, Wang Z, Dong F, Wang F. Induced pluripotent stem cells from goat fibroblasts. Mol. Reprod. Dev. 2013; 80 (12): 1009 - 17

19. Pfannkuche K, Fatima A, Gupta MK, Dieterich R, Hescheler J Initial Colony Morphology-Based Selection for iPS Cells Derived from Adult Fibroblasts Is Substantially Improved by Temporary UTF1-Based Selection. PLoS ONE. 2010; 5(3): e9580.

20. Brown RD, Ambler SK, Mitchell MD, Long CS. The cardiac fibroblast: therapeutic target in myocardial remodeling and failure. Annu Rev Pharmacol Toxicol. 2005; 45: 657 - 87.

21. Souders CA, Bowers SLK, Baudino TA. Cardiac fibroblast: The renaissance cell. Circ Res. 2009; 105 (12):1164 - 76.

22. Husse B, Briest W, Homagk L, Isenberg G, Gekle M. Cyclica mechanical stretch modulates expression of collagen I and collagen III by PKC and tyrosine kinase in cardiac fibroblasts. Am J Physiol Regul Integr Comp Physiol. 2007; 293 (5): R1898 - 907.

23. Santiago J, Dangerfield AL, Rattan SG, Bathe KL, Cunnington RH, Raizman JE, Bedosky K M, Freed D H, Kardami E, Dixon IMC. Cardiac fibroblast to myofibroblast differentiation in vivo and in vitro: expression of focal adhesion components in neonata and adult rat ventricular myofibroblasts. Developmental Dynamics. 2010; 239 (6):1573 - 84

24. Zhang L, Guo J, Zhang P, Xiong Q, Wu SC, Xia L, Roy SS, Tolar J, O'Connell TD, Kyba M, Liao K, Zhang J. Derivation and high engraftment of patient-specific cardiomyocyte sheet using induced pluripotent stem cells generated from adult cardiac fibroblast. Circ Heart Fail. 2015; 8 (1): 156 - 66

25. Yu J, Vodyanik MA, Smuga-Otto K, Antosiewicz-Bourget J, Frane JL, Tian S, Nie J, Jonsdottir GA, Ruotti V, Stewart R, Slukvin II, Thomson J A. Induced pluripotent stem cell lines derived from human somatic cells. Science. 2007; 318 (5858) $1917-20$.

26. Sandmaier SE, Nandal A, Powell A, Garrett W, Blomberg L, Donovan DM, Talbot N, Telugu BP. Generation of induced pluripotent stem cells from domestic goats. Mol Reprod Dev. 2015; 82(9): 709 - 21

27. Loh Y-H, Yang JC, De Los Angeles A, Guo C, Cherry A, Rossi DJ, Park IH, Daley GQ. Excision of a viral reprogramming cassette by delivery of synthetic Cre mRNA. Curr Protoc Stem Cell Biol. 2012; 21(1): 4A - 5

28. Kaji K, Norrby K, Paca A, Mileikovsky M, Mohseni P, Woltjen K. Virus-free induction of pluripotency and subsequent excision of reprogramming factors. Nature. 2009; 458 (7239): 771 - 75.

29. Woltjen K, Michael IP, Mohseni P, Desai R, Mileikovsky M Hämäläinen R, Cowling R, Wang W, Liu P, Gertsenstein M, Kaji K, Sung H, Nagy A. piggyBac transposition reprograms fibroblasts to induced pluripotent stem cells. Nature. 2009; 458 (7239): 766 - 70.

30. Mitra R, Fain-Thornton J, Craig NL. piggyBac can bypass DNA synthesis during cut and paste transposition. EMBO J. 2008; 27 (7): $1097-109$

31. Lin YC, Kuo KK, Wuputra K, Lin SH, Ku CC, Yang YH, Dianfu $\mathrm{Li}$, Wang D, Cao K. Bovine induced pluripotent stem cells are more resistant to apoptosis than testicular cells in response to mono-(2-ethylhexyl) phthalate. Int J Mol Sci. 2014; 15 (3): 5011 31

32. Sumer H, Liu J, Malaver-Ortega LF, Lim ML, Khodadadi K, Verma PJ. NANOG is a key factor for induction of pluripotency in bovine adult fibroblasts. J Anim Sci. 2011; 89 (9): 2708 - 16 
33. Osteil P, Tapponnier Y, Markossian S, Godet M, SchmaltzPanneau B, Jouneau L, Cabau C, Joly T, Blache` re T, Go'cza E, Bernat A, Yerle M, Acloque H, Hidot S, Bosze Z, Duranthon V, Savatier P, Afanassieff M. Induced pluripotent stem cells derived from rabbits exhibit some characteristics of nai"ve pluripotency. Biology Open. 2013; 2 (6): 613 - 28.

34. Yan L, Lei L, Yang C, Gao Z, Lei A, Ma X, Dou Z. Isolation and cultivation of goat embryo stem cells. Sheng Wu Gong Cheng Xue Bao (Chinese journal of biotechnology). 2008; 24 (9): 1670 - 6.

35. Rajala K, Hakala H, Panula S, Aivio S, Pihlajamäki H, Suuronen R, Hovatta O, Skottman H. Testing of nine different xeno-free culture media for human embryonic stem cell cultures. Hum Reprod. 2007; 22(5):1231-8.

36. Blin G, Nury D, Stefanovic S, Neri T, Guillevic O, Brinon B, Bellamy V, Rücker-Martin C, Barbry P, Bel A, Bruneval P, Cowan C, Pouly J, Mitalipov S, Gouadon E, Binder P, Hagège A, Desnos M, Renaud JF, Menasché P, Pucéat M. A purified population of multipotent cardiovascular progenitors derived from primate pluripotent stem cells engrafts in postmyocardial infarcted nonhuman primates. J Clin Invest. 2010; 120 (4):1125-39.

37. Brimble SN, Sherrer ES, Uhl EW, Wang E, Kelly S, Merrill AH Jr, Robins AJ, Schulz TC. The cell surface glycosphingolipids SSEA-3 and SSEA-4 are not essential for human ESC pluripotency. Stem Cells. 2007; 25(1): 54 - 62.

38. Takahashi S, Kobayashi S, Hiratani I. Epigenetic differences between naïve and primed pluripotent stem cells .Cell Mol Life Sci. 2018; 75(7):1191 - 1203.

39. Park JK, Kim HS, Uh KJ, Choi KH, Kim HM, Lee T, Yang BC, Kim HJ, Ka HH, Kim H, Lee CK. Primed Pluripotent Cell Lines Derived from Various Embryonic Origins and Somatic Cells in Pig. PLoS One. 2013; 8(1): e52481.

40. Fujishiro SH, Nakano K, Mizukami Y, Azami T, Arai Y, Matsunari $\mathrm{H}$, Ishino R, Nishimura T, Watanabe M, Abe T, Furukawa Y, Umeyama K, Yamanaka S, Ema M, Nagashima H, Hanazono Y. Generation of naive-like porcine-induced pluripotent stem cells capable of contributing to embryonic and fetal development. Stem Cells Dev. 2013; 22(3):473-82.

41. Greber B, Lehrach H, Adjaye J. Fibroblast growth factor 2 modulates transforming growth factor $b$ signaling in mouse embryonic fibroblasts and human ESCs (hESCs) to support hESC self-renewal. Stem Cells. 2007; 25 (2):455- 64.
42. Ono T, Suzuki Y, Kato Y, Fujita R, Araki T, Yamashita T, Kato H, Torii R, Sato N. A single-cell and feeder-free culture system for monkey embryonic stem cells. PLoS One. 2014; 9(2): e88346.

43. Gallego Romero I, Pavlovic BJ, Hernando-Herraez I, Zhou X, Ward MC, Banovich NE, Kagan CL, Burnett JE, Huang CH, Mitrano A, Chavarria CI, Ben-Nun IF, Li Y, Sabatini K, Leonardo TR, Parast M, Marques-Bonet T, Laurent LC, Loring JF, Gilad Y. A panel of induced pluripotent stem cells from chimpanzees: a resource for comparative functional genomics. eLife. 2015; 4: e07103.

44. Huang B, Li T, Alonso-Gonzalez L, Gorre R, Keatley S, Green A, Turner P, Kallingappa P K, Verma V, Oback B. A virus-free polypromoter vector induces pluripotency in quiescent bovine cells under chemically defined conditions of dual kinase inhibition. PloS one. 2011; 6 (9): e24501.

45. Pyle AD, Lock LF, Donovan PJ. Neurotrophins mediate human embryonic stem cell survival. Nat. Biotechnol. 2006; 24 (3): 344 50 .

46. Müller T, Fleischmann G, Eildermann K, Mätz-Rensing K, Horn P A, Sasaki E, Behr R. A novel embryonic stem cell line derived from the common marmoset monkey (Callithrix jacchus) exhibiting germ cell-like characteristics. Human Reproduction. 2009; 24(6): 1359 - 72 .

47. Pieters T, Goossens S, Haenebalcke L, Andries V, Stryjewska A, De Rycke R, Lemeire K, Hochepied T, Huylebroeck D, Berx G, Stemmler MP, Wirth D, Haigh JJ, van Hengel J, van Roy F. p120 Catenin-Mediated Stabilization of E-Cadherin Is Essential for Primitive Endoderm Specification. PLoS Genet. 2016; 12(8): e1006243.

48. Khoo MLM, McQuade LR, Smith MSR, Lees JG, Sidhu KS, Tuch BE. Growth and Differentiation of Embryoid Bodies Derived from Human Embryonic Stem Cells: Effect of Glucose and Basic Fibroblast Growth Factor1. Biology of Reproduction. 2005; 73(6), 1147-56.

49. Yasuda E, Seki Y, Higuchi T, Nakashima F, Noda T, Kurosawa H. Development of cystic embryoid bodies with visceral yolk-sac-like structures from mouse embryonic stem cells using low-adherence 96-well plate. J Biosci Bioeng. 2009; 107(4):442-6.

50. Sivasubramaiyan K, Totey S, Bhat V, Totey SM, Deb K. Y-27632 enhances differentiation of blastocyst like cystic human embryoid bodies to endocrinologically active trophoblast cells on a biomimetic platform. J Biomed Sci. 2009; 16 (1):88.

\section{Abbreviations}

\begin{tabular}{|c|c|}
\hline$\alpha \mathrm{SMA}$ & : $\alpha$ Smooth muscle actin. \\
\hline bFGF & : b- Fibroblast growth factor. \\
\hline c-MYC & : Myelocytomatosis Oncogene. \\
\hline DAPI & : 4', 6-Diamidino-2-phenylindole is a fluorescent stain that binds strongly to A-T rich regions in DNA. \\
\hline DDR2 & : Discoidin Domain Receptor 2. \\
\hline DMEM & : Dulbecco's Modified Eagle's medium. \\
\hline DPBS & : Dulbecco's Phosphate-Buffered Saline. \\
\hline FBS & : Fetal bovine serum. \\
\hline GCF & : Goat cardiac fibroblasts. \\
\hline GFP & : Green fluorescent protein. \\
\hline GiPSC & : Goat induced pluripotent stem cells after removal of transposons. \\
\hline hiPSCs & : Human induced pluripotent stem cells. \\
\hline iPSCs & : Induced pluripotent stem cells. \\
\hline KLF4 & : Krüppel-like factor 4 \\
\hline KOSR & : Knockout serum replacement. \\
\hline LIF & : Leukemia inhibitory factor. \\
\hline LIN28 & : Lin-28 homolog $\mathrm{A}$ is a protein that in humans is encoded by the LIN28 gene. \\
\hline
\end{tabular}




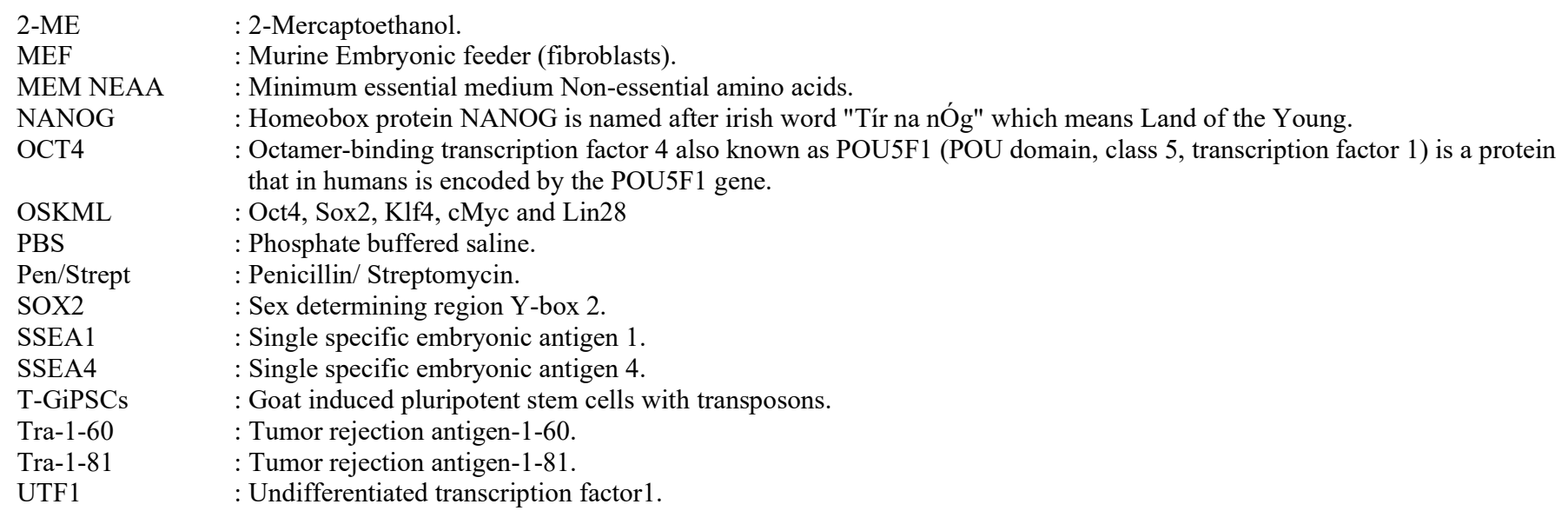

\section{Potential Conflicts of Interests}

None

\section{Acknowledgement:}

My profound gratitude is due to to Pr. Dr. med. Konrad Brockmeier, head of pediatric cardiology department, Cologne University, who honored me by being my Professor. My thanks and gratitude are for his great support throughout the course of this study. I am greatly indebted to my supervisor PD Dr. Kurt Pfannkuche, neurophysiology department, Cologne University, who honored me by giving me the opportunity to be part of his group, learn from his expanded experience. I am greatly honored to express my endless thanks to him, for his productive guidance, and encouragement to finish this work. I would like to express my deep appreciation and thankfulness to my Colleagues in the group and in the whole institute especially Mr. Daniel Derichsweiler and Ms. Annette Köster.

\section{Sponsors/Grants}

This work was supported by the grant from BMBF-2014 project to PD Dr. Kurt Pfannkuche, Institute of Neurophysiology, University of Cologne, and our Collaborative partner institute Sindh Agriculture University Tandojam Pakistan. The research was done through GERLS long term scholarship from Deutscher Akademischer Austauschdienst (DAAD) (Head office address: Kennedyallee 50, D-53175 Bonn, Germany; Cairo office address: 11 El-Saleh Ayoub, Al Gabalaya, Zamalek, Cairo 11211, Egypt).

\section{Additional Information}

Supplementary Information accompanies this article. Supplementary information is linked to the online version of the article.

\section{Corresponding Author}

Mira Barsoum Nashid Barsoum Hanna, Faculty of medicine (Kasr El-Aini) Cairo University, El-Maniel, Cairo 11451, Egypt E mail ID: mirabarsoumhanna@gmail.com / ursmortadella@yahoo.com / mira.b.hanna@kasralainy.edu.eg 